\section{Advances in Management of Hepatocellular Carcinoma}

\section{ABSTRACT 1}

Gastroenterology 2016;150:488-498.

Hepatic Arterial Infusion of Low-Density Lipoprotein Docosahexaenoic Acid Nanoparticles Selectively Disrupts Redox Balance in Hepatoma Cells and Reduces Growth of Orthotopic Liver Tumors in Rats Xiaodong Wen, ${ }^{1}$ Lacy Reynolds, ${ }^{1}$ Rohit S. Mulik, ${ }^{1}$ Soo Young Kim, ${ }^{1}$ Tim Van Treuren, ${ }^{1}$ Liem H. Nguyen, ${ }^{2,3}$ Hao Zhu, $^{2,3}$ and Ian R. Corbin ${ }^{1,3}$

${ }^{1}$ Advanced Imaging Research Center; ${ }^{2}$ Children's Research Institute, Department of Pediatrics and Internal Medicine, Simmons Comprehensive Cancer Center, Center for Regenerative Science and Medicine; and ${ }^{3}$ Internal Medicine Division of Liver and Digestive Diseases, University of Texas Southwestern Medical Center, Dallas, Texas, USA

Background \& aims: Dietary intake of the natural omega-3 fatty acid docosahexaenoic acid (DHA) has been implicated in protecting patients with viral hepatitis $\mathrm{B}$ or $\mathrm{C}$ from developing hepatocellular carcinoma (HCC). Little is known about the effects of DHA on established solid tumors. Here we describe a low-density lipoprotein based nanoparticle that acts as a transporter for unesterified DHA (LDL-DHA) and demonstrates selective cytotoxicity toward HCC cells. We investigated the ability of LDL-DHA to reduce growth of orthotopic hepatomas in rats.

Methods: AxC-Irish (ACI) rats were given intrahepatic injections of rat hepatoma cells (H4IIE); 24 tumor-bearing rats (mean tumor diameter, $1 \mathrm{~cm}$ ) were subject to a single hepatic artery injection of LDL nanoparticles $(2 \mathrm{mg} / \mathrm{kg})$ loaded with DHA (LDL-DHA), triolein (LDL-TO), or sham surgery controls. Tumor growth was measured by magnetic resonance imaging and other methods; tumor, liver, and serum samples were collected and assessed by histochemical, immunofluorescence, biochemical, and immunoblot analyses.

Results: Three days after administration of LDL-TO or sham surgery, the control rats had large, highly vascularized tumors that contained proliferating cells. However, rats given LDL-DHA had smaller, pale tumors that were devoid of vascular supply and $>80 \%$ of the tumor tissue was necrotic. Four to 6 days after injection of LDL-DHA, the tumors were 3 -fold smaller than those of control rats. The liver tissue that surrounded the tumors showed no histologic or biochemical evidence of injury. Injection of LDL-DHA into the hepatic artery of rats selectively

http://dx.doi.org/10.1016/j.jceh.2016.03.006 deregulated redox reactions in tumor tissues by increasing levels of reactive oxygen species and lipid peroxidation, depleting and oxidizing glutathione and nicotinamide adenine dinucleotide phosphate, and significantly down-regulating the antioxidant enzyme glutathione peroxidase-4. Remarkably, the redox balance in the surrounding liver was not disrupted.

Conclusion: LDL-DHA nanoparticle selectively kills hepatoma cells and reduces growth of orthotopic liver tumors in rats. It induces tumor-specific necrosis by selectively disrupting redox balance within the cancer cell.

\section{ABSTRACT 2}

International Journal of Radiation Oncology•Biology•Physics (10.1016/j.ijrobp.2016.02.027)

A Randomized Clinical Trial Comparing Proton Beam Radiotherapy to Transarterial Chemoembolization for Hepatocellular Carcinoma-Results of an Interim Analysis

David A. Bush, ${ }^{1}$ Jason C. Smith, ${ }^{2}$ Jerry D. Slater, ${ }^{1}$ Michael L. Volk, ${ }^{3}$ Mark E. Reeves, ${ }^{4}$ Jason Cheng, ${ }^{3}$ Roger Grove, ${ }^{1}$ and Michael E. de Vera, ${ }^{3}$

${ }^{1}$ Loma Linda University Medical Center, Department of Radiation Medicine, ${ }^{2}$ Department of Diagnostic Radiology, ${ }^{3}$ Transplantation Institute and Liver Center, ${ }^{4} \mathrm{~V}$ A Loma Linda Health Care System 11201 Benton Street Loma Linda, CA 92357, USA

Objective: This prospective randomized clinical trial was developed to compare treatment outcomes among patients with newly diagnosed hepatocellular carcinoma (HCC). This report describes results of a planned interim analysis.

Methods: Eligible subjects had either clinical or pathologic diagnosis of HCC and met either Milan or San Francisco transplant criteria. Patients were randomly assigned to transarterial chemoembolization (TACE) or to proton beam radiotherapy. Patients randomized to TACE received at least one TACE with additional TACE for persistent disease. Proton beam radiotherapy was delivered to all areas of gross disease to a total dose of $70.2 \mathrm{~Gy}$ in 15 daily fractions over 3 weeks. Primary endpoint was progression-free survival, with secondary endpoints of overall survival, local tumor control and treatment-related toxicities as represented by post-treatment days of hospitalization.

Results: At the time of this analysis 69 subjects were available for analysis. 36 were randomized to TACE and 33 to proton. Total days of hospitalization within 30 days of TACE/proton was 166 and 24 days respectively $(p<0.001)$. Ten TACE and 12 proton patients underwent liver transplantation following treatment. Viable tumor identified in the explanted livers following TACE/proton averaged 2.4 and $0.9 \mathrm{~cm}$ respectively. Pathologic complete response following TACE/proton was $10 \% / 25 \%(p=0.38)$. The 2 -year overall survival for all patients was $59 \%$ with no difference 
between treatment groups. Median survival time was 30 months (95\% CI - 20.7-39.3 months). There was a trend toward improved 2-year local tumor control (88\% vs. $45 \%$, $p=0.06)$ and progression-free survival (48\% vs. $31 \%$, $p=0.06$ ) favoring the proton beam treatment group.

Conclusion: This interim analysis indicates similar overall survival rates for proton beam radiotherapy and TACE. There is a trend toward improved local tumor control and progression-free survival with proton beam. There are significantly fewer hospitalization days following proton treatment that may indicate reduced toxicity with proton beam therapy.

\section{COMMENTS}

Advances in technologies are of interest to treating physicians-whether it is the development of new drugs as oral directly acting agents (DAAs) for hepatitis C, or development of new techniques for delivery of drugs, especially those acting on targets of interest. These advances are expected to improve the management of patients in terms of improved outcomes and reduced potential systemic side effects. The future therapies are expected to be individualized and 'tailor-based', which have the advantages of being focused and associated with the best response for a particular phenotype, although the higher costs are a deterrent. The cost is expected to come down gradually with time, as has been the story of oral DAAs in India and across the globe.

Hepatocellular carcinoma (HCC) is an important cause of cancer and mortality across the world. A recent large representative survey from India reported liver cancer to be the 4th most common cause of cancer-related deaths in males, and 8th most common cause in females. ${ }^{1}$, The incidence of HCC and associated mortality are increasing across the world, and India is no exception. Therapeutic options are limited for patients who present in intermediate and advanced stages, which accounts for the majority of clinically evaluated patients. The options include transarterial chemotherapy and Sorafenib-which is an oral multikinase inhibitor and the only therapy associated with improved survival in patients with advanced HCC. ${ }^{2}$, Therefore, there is an unmet need for the development of therapeutic modalities for the management of HCC. In a recent publication in Gastroenterology, Wen et al. ${ }^{3}$, explored the role of low-density lipoprotein docosahexaenoic acid labeled (LDL-DHA) nanoparticles in the management of HCC in rats. This study was based on the principle of antitumor effect of omega-3-fatty acids in patients with hepatitis $\mathrm{B}$ or $\mathrm{C}$ infection. ${ }^{4}$ In a stepwise manner, the authors have reported the mechanism of action of these LDL-DHA nanoparticles.

In the first step, LDL-DHA nanoparticles were delivered to AXI rats containing hepatomas through the hepatic artery. Beautifully, it has been demonstrated that the particles got deposited only in tumor tissue and resulted in a significant reduction of the tumor size. In contrast to transarterial chemoembolization (TACE)-in which gel foam embolisation of feeding vessels is done, which results in necrosis of normal hepatic parenchyma along with cancer-these nanoparticles are non-embolic and do not affect the normal hepatocytes as shown by the absence of a rise in hepatic transaminase levels. However, certain questions still need to be answered, namely-do the tumor cells of hepatocellular carcinoma have LDL receptors and are they specifically upregulated for uptake of LDL containing docosahexaenoic acid?

The second step involved deciphering the mechanism of action of these LDL-DHA nanoparticles. The authors have shown that LDL-DHA particles can induce tumor cell death by free radicals inducing a redox stress. In this simple rat study, they have been able to demonstrate two important findings, ${ }^{1,}$ LDL-DHA nanoparticles get localized to tumor cells only and ${ }^{2}$ they are tumoricidal at concentrations which are much lower than the toxic doses for normal hepatocytes. These two properties firmly support further evaluation using clinical trials involving human subjects, which will be the next logical step.

In addition, fluorescent labeled LDL-DHA nanoparticles can be utilized for intra-operative localization of tumor during hepatic resection. Another potential utility is in metastatic HCC, where they may be useful in the management of the extrahepatic burden of the tumor. In future, it may prove worthwhile to explore the tumoricidal role of w-3 fatty acid in other malignancies as well. The results appear promising and may bring about a significant change in the management of HCC in the future.

HCC is generally considered to be a radioresistant tumor; this concept arose from the poor outcomes of use of radiotherapy in these tumors reported in initial studies. A major drawback of these studies was either insufficient doses of radiation, or radiation given to the whole liver. Contrary to this belief (of lack of response of HCC to radiotherapy), current evidence suggests that the radiosensitivity of HCC is similar to epithelial tumors. There have been substantial advances over the past decade in the delivery of radiation therapy. Three-dimensional conformal RT (3D-CRT) delivers localized radiation three-dimensionally using computer softwares, thus reducing the potential side effects of radiation to normal tissues. The dose distribution can also be modulated as required. Particle beams (using protons) have the advantage of greater levels of ionization per unit length. Another advantage is that they specifically deliver energy to localized areas. Bush et al. in a randomized trial compared proton beam radiotherapy with TACE. In this interim analysis so far 69 patients had been recruited (33 in proton therapy and 36 in TACE). ${ }^{5}$ The baseline characteristics were similar in both groups. Multiple sessions of both TACE and proton therapy were used. The total days of hospital stay were significantly less in the proton therapy 
group as compared with the TACE group. There were no significant differences in the pathologic complete response, overall survival and survival after liver transplant between the two groups. Although, as this is an interim analysis, the results of the complete trial will show more clear data regarding the outcomes. The current results show that proton beam therapy may be an additional therapeutic option for HCC. The results of combining proton beam therapy with other therapies for HCC should be interesting. This therapy needs further exploration in management of HCC.

In conclusion, there have been significant recent advances in the understanding of molecular pathogenesis of HCC and therapeutic options available. It remains to be seen how these advances translate into clinical benefits in terms of survival and reducing tumor burden.

\section{REFERENCES}

1. Dikshit R, Gupta PC, Ramasundarahettige C, et al. Cancer mortality in India: a nationally representative survey. Lancet. 2012;379 (9828):1807-1816.
2. Llovet JM, Ricci S, Mazzaferro V, et al. Sorafenib in advanced hepatocellular carcinoma. N Engl J Med. 2008;359(4):378-390.

3. Wen X, Reynolds L, Mulik RS, et al. Hepatic arterial infusion of lowdensity lipoprotein docosahexaenoic acid nanoparticles selectively disrupts redox balance in hepatoma cells and reduces growth of orthotopic liver tumors in rats. Gastroenterology. 2016;150(2): 488-498.

4. Sawada N, Inoue M, Iwasaki M, et al. Consumption of n-3 fatty acids and fish reduces risk of hepatocellular carcinoma. Gastroenterology. 2012;142(7):1468-1475.

5. Bush DA, Smith JC, Slater JD, et al. A randomized clinical trial comparing proton beam radiotherapy to transarterial chemoembolization for hepatocellular carcinoma-results of an interim analysis. Int J Radiat Oncol Biol Phys. 2016. http://dx.doi.org/10.1016/ j.ijrobp.2016.02.027.

Sawan Bopanna, Shalimar Department of Gastroenterology, All India Institute of Medical Sciences, New Delhi, India

*Address for correspondence: Shalimar, Department of Gastroenterology, All India Institute of Medical Sciences, New Delhi, India. Tel.: +919968405815. E-mail: drshalimar@yahoo.com 